In Proc. of 10th IFIP International Conference on Personal Wireless Communications/ PWC'05, Colmar, France, August 2005

\title{
ADAPTIVE SCHEDULING FOR HETEROGENEOUS TRAFFIC FLOWS IN CELLULAR WIRELESS OFDM-FDMA SYSTEMS*
}

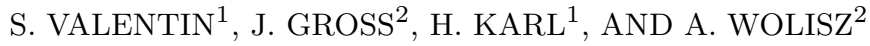 \\ ${ }^{1}$ University of Paderborn, \\ Warburger Straße 100, \\ 33098 Paderborn, Germany \\ E-mail: \{stefan.valentin| holger.karl\}@upb.de \\ ${ }^{2} T U$ Berlin, \\ Einsteinufer 25, \\ 10587 Berlin, Germany, \\ E-mail: \{gross | wolisz\}@tkn.tu-berlin.de
}

\begin{abstract}
In this paper we study the performance gain achieved by introducing dynamic subcarrier scheduling schemes to OFDM systems in the presence of heterogeneous traffic streams. Dynamic subcarrier scheduling copes with channel state variation by dynamically assigning different subcarriers to terminals, e.g. in the downlink of a cellular system. In addition to the channel state variations, also the arrival of data is highly variable for most applications. This calls for schemes that exploit both sources of variation. In the homogeneous traffic scenario, dynamic subcarrier scheduling in combination with semantic queueing mechanisms can significantly increase the capacity of an OFDM cell. In a heterogeneous traffic scenario, however, these mechanisms do not simply carry over. Therefore, in this work we study first the performance increase achieved for heterogeneous flows by purely switching from static to dynamic subcarrier scheduling and then introduce a semantic traffic management scheme that improves the achieved performance further by sacrificing the transmission of semantically unimportant video packets for important video packets and for packets related to HTTP.
\end{abstract}

\footnotetext{
*The main part of this work has been done while the authors were with the TU Berlin. It has been partially supported by the German research funding agency 'Deutsche Forschungsgemeinschaft (DFG)' under the program 'Adaptability in Heterogeneous Communication Networks with Wireless Access (AKOM)'.
} 


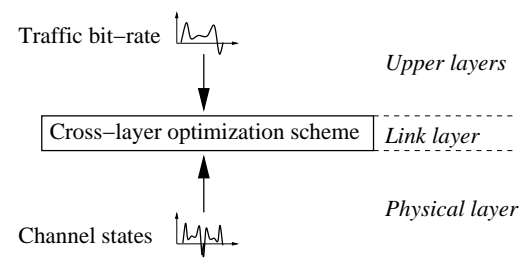

Figure 1. Sources of variability and the related layers

\section{Introduction}

Delivering high quality multimedia streams via a wireless link is still a challenging task for mobile communication systems. In addition to high transmission speed, quality aspects, such as bounded delays, are required for the transmission. In wireless systems multi-path propagation and movement leads to Inter-Symbol Interference (ISI) and fading. ISI can be minimized by modern modulation schemes, e.g. Orthogonal Frequency Division Multiplexing (OFDM), where the spectrum is separated into narrow bands, the so-called subcarriers. Fading, however, leads to drastic changes in signal attenuation and Symbol Error Probability (SEP) and cannot be neutralized on the physical layer (PHY). Thus, the available channel resource is a random variable with rather large dynamics. In addition, the resource (bit rate) demanded by the packet streams, which have to be sent by the transmission system, varies. This variation is common in packet-switched networks and depends on traffic characteristics which are originally defined at the sender's Application layer (APP).

In multi-terminal environments these random processes - channel and traffic variation - add new challenges for the management of the channel resources. With static scheduling both fluctuations lead automatically to over- or under-provisioning of the channel resources per packet stream. A solution is the dynamic distribution of resources according to the wireless channel and traffic stream parameters. This adaption can be done in the APP, e.g. by the video codec, as well as in lower layers. Performing the adaptive scheduling on the Logical Link Control layer (LLC) enables fast access to the information provided by the lower and higher layers, e.g. PHY and APP. As illustrated in Figure 1, this so-called cross-layer optimization approach adapts the Medium Access Control (MAC) to the two sources of variability, traffic bit rate and channel state. In this paper we discuss the cross-layer optimization scheme separately for each source of variability. The first discussed component is called dynamic subcarrier scheduling. It 
follows the channel-state dependent scheduling approach [1] and distributes the available capacity (e.g. OFDM subcarrier) to the terminals according to the measured channel state. The second component, called traffic management [2], controls the bit rate of the source according to application layer parameters. We discuss both optimization schemes in greater detail in Section 3.

Performance studies were done in previous work for both schemes considering only one type of application layer traffic. While dynamic subcarrier scheduling was typically studied for bulk or HTTP traffic [3], for traffic management streaming media is relevant [4]. In these homogeneous traffic scenarios each type of APP traffic is considered separately, which clearly does not reflect the nature of the Internet. Here, typically a combination of HTTP, streaming, and bulk related traffic flows is present [5]. Due to the different demands of the flow types on the transmission system (e.g. Quality of Service (QoS) guarantees for streaming, fault-tolerant and fast transmission for HTTP) especially the combination of HTTP and video streaming is interesting. For cross-layer optimization approaches in wireless systems this combination has not been investigated so far.

For this so-called heterogeneous traffic scenario we first study how the combination of the different traffic characteristics affects the performance of the cross-layer optimization scheme. Second, we propose a new traffic management scheme, called Video Queue Management (VQM), which executes a filter that removes video data from the access point queue. While the filter considers semantic parameters in order to minimize the quality decrease due to packet removal, parameters from the link and the physical layer are evaluated for load estimation.

The model of the considered dynamic OFDM-FDMA system is discussed in the next section of this paper. Section 3 introduces the basic approach and algorithms of VQM. Here the two major parts - load estimation and video filtering - are discussed in greater detail. In Section 4, the results of the performance study for dynamic subcarrier scheduling and the proposed traffic management scheme are presented. In the last section, we conclude the paper.

\section{System model}

We consider a single wireless cell of radius $R$ in which a certain number of $J$ terminals as well as one access point are located. The terminals are connected via a radio link to the access point, which controls all wireless 
transmission. Since the optimization of the uplink is not the topic of this paper only the downlink transmission is investigated. Data is transmitted to the terminals using the available bandwidth $B$ around the center frequency $f_{c}$. Typical effects which occur during the terrestrial transmission of radio signals are considered in the system model. Specifically, path loss and, due to multi-path propagation, frequency-selective fading are modeled. The terminals are independently moving within the cell. Due to this movement, also time-selective fading occurs. The OFDM transmission scheme separates $B$ into $S$ subcarriers. Each symbol is transmitted during the symbol time $T_{s}$ using constant transmission power $P_{t x}$ per subcarrier. An adaptive modulation scheme selects one modulation type out of five (BPSK, QPSK, 16-QAM, 64-QAM, and 256-QAM) in order to stay below the SEP bound $P_{s}$.

Subsets of subcarriers are distributed to the terminals using a static or dynamic Frequency Division Multiple Access (FDMA) scheme. The dynamic subcarrier scheduling scheme, applied in case of dynamic FDMA, is discussed more detailed in Section 3. The subcarrier assignments are valid for one MAC cycle of length $T_{f}$. The terminals in the cell are receiving data flows from external sources that are connected to the access point via a cable link. The flows consist of packets and are queued at the access point until they are forwarded to the terminals via the wireless link. At the access point transmission functions and queueing are handled for each terminal separately. A fraction of the flows is related to Web pages. All other packets are related to MPEG- 4 coded, Variable Bit Rate (VBR) video streams. The Web pages are transmitted using TCP/IP, for the video streams UDP/IP is employed. For the video streams a traffic management scheme is used in the link layer at the access point. We will give an overview of this scheme in the next section.

\section{Optimization Approach}

\subsection{Dynamic subcarrier scheduling}

Since channel-state dependent scheduling was proposed by Bhagwat et al. [1] several scheduling algorithms are under discussion. Performance studies [6] have shown that the proportionally fair and the exponential rule are good candidates for the support of real-time and non-real-time applications with high data rates. However, all these algorithms consider an optimal solution for the subcarrier assignment problem, an optimization problem typically considering the parameters link state and queueing time. In scenarios with 
many terminals the calculation time for the optimal solution will exceed the assignment cycle (i.e. MAC-frame), which is typically short in high data rate scenarios.

The dynamic subcarrier scheduling scheme employed in this paper, called advanced Dynamic Algorithm (aDA), belongs to the class of proportionally fair scheduling algorithms. It provides a slightly suboptimal but fast solution for the assignment problem [7] depending on the most recent subcarrier states. With the state of each subcarrier, which depends on the chosen modulation type, aDA selects subcarriers with the highest possible states. This selection of subcarriers according to channel states and the further adaption of the number of subcarriers to the queue length leads to a continuous variation of the downlink capacity per terminal.

\subsection{Traffic management for video streams}

For the transmission of videos in real-time application scenarios, such as video conferences or the broadcasting of live events, small, predictable transmission delays are required. This is even the case if the state of the wireless channel is temporarily bad or the sender rate is temporarily high. For the considered system and the chosen optimization approach this means that the queueing delay at the access point should be minimized for each packet. It is beneficial if the scheduler is aware of application layer information regarding the packets on the link layer. With this knowledge those video-related packets whose transmission time has exceeded an estimated deadline $D$ can be removed from the queue.

The temporal compression of modern video codecs leads to three different types of video frames. While the so called $I$-frame contains a whole (spatial compressed) video picture, $P$ - and $B$-frames contain only temporal differences to the preceding frames (P-frame) or to the preceding and following frames (B-frame). Since I-frames have the highest entropy and B-frames contain the least amount of information, the deadline of the video packets can be weighted according to the semantic relevance of the frame type. One example is the so-called semantic-aware traffic management scheme [8]. Here I-frames are only removed from the queue after the transmission delay for the related packets has exceeded the full deadline $D$. B-frames are removed first, followed by P-frames.

In this paper we propose an extension to the semantic-aware scheme that is called Video Queue Management (VQM). With VQM an additional

removal cycle, called packet filter, is invoked if the traffic load exceeds the 


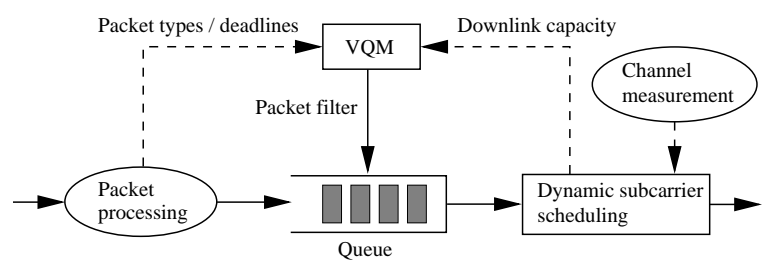

Figure 2. General architecture of the considered dynamic MAC scheme

threshold $\alpha$ after the semantic-aware scheduling is performed. The traffic load is estimated once per MAC cycle $n$ by the ratio $R(n)=\frac{s(n)}{F(n-1)}$, where $F$ stands for the downlink capacity, which varies with dynamic subcarrier scheduling, and $s$ for the sum of bits already removed by the semantic-aware traffic management scheme. Since the traffic management scheme has to be performed before dynamic subcarrier scheduling, the value $F(n-1)$ of the preceding downlink phase is used. The idea to use $R(n)$ as a representation of the system load is, that $s$ increases with rising system load due to more missed deadlines or that the capacity $F$ decreases. The packet filter removes whole packets from the access point queue and considers semantic features in order to minimize the additional distortion of the video. Only B-frame related packets are removed during the iteration of the packet filter over the whole queue. The filter is executed until the sum of the bits removed reaches a certain quality threshold $\beta$.

The resulting combined cross-layer optimization scheme is illustrated in Figure 2. All components denoted by the blocks rely on cross-layer information, which is extracted by the functions marked by the ellipses. The provision of the extracted data to the optimization schemes is illustrated by the dashed arrows. Dynamic subcarrier scheduling considers channel states for its scheduling decision. VQM relies on the scheduling decision and on semantic features extracted from the video stream.

\section{Performance study}

\subsection{Metrics and methodology}

Two types of traffic are considered to be transmitted via the wireless link: Web pages and MPEG-4 coded video streams. On the user level both traffic types are differently affected by LLC capacity variation. Thus, we use two metrics, one for each traffic type. For the transmission of Web pages 
the latency per page is measured on the application layer of each receiver. The quality of the transmitted video is measured using the Distortion In Interval (DIV) metric [8]. This metric provides an approximation of the subjective Mean Opinion Score (MOS) grade by using the Peak Signal-toNoise Ratio (PSNR) value, which can be calculated for each video frame. PSNR and MOS are widely used for the evaluation of picture quality. The video quality is rated to be unacceptable if more than $20 \%$ of the decoded video pictures in a $20 \mathrm{~s}$ interval have a lower MOS grade than the original pictures [8]. Note that this threshold is somewhat subjective, it could also be put at $18 \%$ or $22 \%$, but definitely not at values like $40 \%$ or $60 \%$.

Both metrics are investigated in the performance study for four different setups. Using the notation subcarrier scheduling/traffic management scheme for each setup, the first setup is called Static/Static. Here no optimization is done at all. Each terminal receives a set of subcarriers at the beginning of the simulation and FIFO is used for the packet queueing. The dynamic assignment of the subcarrier sets is considered in the Dynamic/ Static setup. Then we combine dynamic subcarrier scheduling with traffic management. The performance of the semantic-aware approach in combination with the dynamic subcarrier scheduling is studied in the Dynamic/ Semantic case. Finally, we investigate dynamic subcarrier scheduling together with the proposed VQM scheme in the Dynamic/VQM setup.

For all scenarios and metrics the average of all values measured during the simulated time is shown versus the number of video-receiving terminals. Due to their small size the 0.95-confidence intervals are not shown.

\subsection{Simulation parameterization}

For the simulation we chose the following settings. The total bandwidth of $16.25 \mathrm{MHz}$ in the $5.2 \mathrm{GHz}$ band is separated into 48 subcarriers, which is equivalent to 802.11a. In order to assign at least one subcarrier per terminal $J \leq 48$ terminals are moving with a maximum speed of $1 \mathrm{~m} / \mathrm{s}$ in a cell of radius $R=100 \mathrm{~m}$. Path loss, shadowing and fading are modeled according to the channel model proposed in [9]. The delay spread is set according to the ETSI C large open space model [10] to $0.15 \mu \mathrm{s}$. Data is transmitted using a constant power of $-7 \mathrm{dBm}$. The SEP threshold for the dynamic modulation scheme is $10^{-2}$. For TCP the NewReno flavor and the common maximum segment size of 1460 Bytes is chosen. Since two types of application layer traffic are considered in this paper, $J_{h}$ out of $J$ terminals are receiving Web pages while video streams are simultaneously 


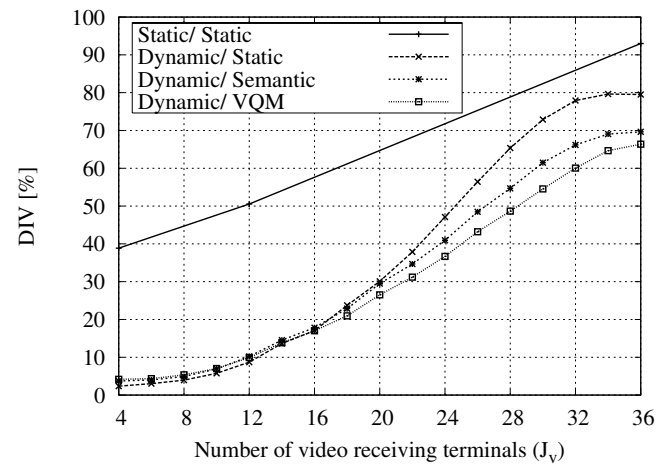

Figure 3. Mean DIV of the video streams for four optimization scheme setups

transmitted to $J_{v}=J-J_{h}$ terminals. In the performance study we set $J_{h}=12$ and $J_{v}$ is varied. The HTTP traffic is modeled according to the corporate environment in [11]. For the mean inter-session time we chose $3 \mathrm{~s}$ to assure an adequate utilization of the system. The MPEG-4 coded video streams have mean bit rate of $r_{v}=951 \mathrm{KBps}$, using the common Group of Pictures (GOP) IBBPBBPBBPBB, which provides good quality on handheld terminals. In the Dynamic setups during each MAC cycle of $2 \mathrm{~ms}$ dynamic subcarrier scheduling and traffic management are performed. The traffic management schemes semantic-aware scheduling and VQM use the (heuristic) deadline $D=100 \mathrm{~ms}$. B-frames are removed at $0.5 D$, P-frames at $0.75 D$ and I-frames at $D$. In addition to these weights VQM makes use of two additional thresholds. The threshold for the load-rate $\alpha$ was chosen to be 5 . For the video quality we chose the threshold $\beta=12$.

\subsection{Simulation results}

In this section we present the results of the performance study of the crosslayer optimization schemes versus increasing numbers of video stream receiving terminals in the cell $\left(J_{v}\right)$. With the metrics shown for the two traffic types better results are achieved for smaller values. Because the overall capacity of the wireless channel is limited, both metrics degrade with increasing $J_{v}$ due to the contention increase of the traffic flows.

As shown in Figure 3 and 4 the dynamic subcarrier scheduling clearly outperforms the static case for both types of traffic. For an upper DIV bound of $20 \%$ gains of up to $62 \%$ can be achieved with the dynamic scheduling compared to the static scheme. This means that up to 17 video streams 


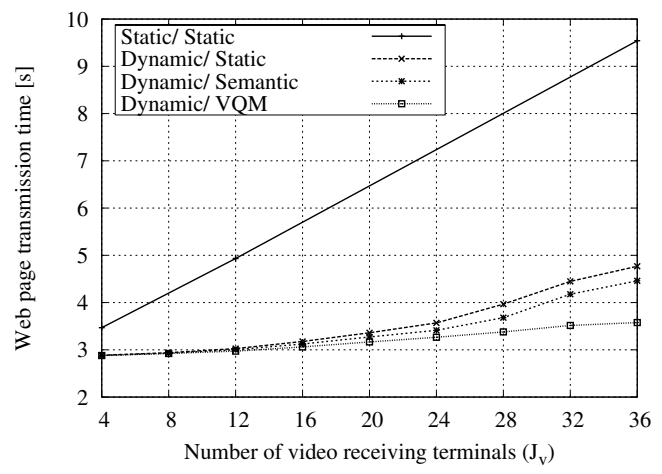

Figure 4. Mean Web page transmission time for four optimization scheme setups

can be sent with dynamic subcarrier scheduling while no stream of this quality can be sent without any optimization scheme. Furthermore, the average transmission time of the simultaneous transmitted Web pages is decreased by up to $47 \%$.

The results for the traffic management scheme show small increases of the video quality near the upper DIV bound at $J_{v}=18$. Compared to the Dynamic/Static setup the semantic-aware scheduling increases the performance gain by only $4 \%$ for video and by $4 \%$ for Web page transmission. With the Dynamic/VQM setup a gain of $12 \%$ for video quality and of $6 \%$ for Web pages can be reached. Furthermore, semantic weighting and the additional filter of VQM do not significantly decrease the video quality.

\section{Conclusions}

In this paper we have investigated the combination of the two cross-layer optimization approaches channel-state dependent scheduling and traffic management for a wireless OFDM-FDMA system in heterogeneous traffic scenarios. We have shown that channel-state dependent scheduling increases the performance even with heterogeneous traffic in the downlink. Here gains of up to $62 \%$ were found for the transmission of MPEG- 4 coded video streams and up to $47 \%$ for the Web page transmission time, respectively. This is comparable to the performance increase found for streaming video in $[8]$.

Furthermore, we have found that traffic management schemes do not lead to a major performance increase in heterogeneous traffic scenarios. Here only a part of the data is related to video streams, whose redun- 
dancy can be exploited by the semantic weighting schemes. Thus, the achieved gains are quite low if compared to the improvements of semanticaware scheduling in homogeneous scenarios [8]. Semantic filtering such as VQM, however, further increases the performance at feasible complexity (one queue iteration). This performance increase rises with the traffic load that can be filtered. This makes VQM applicable in cases of congestion. Here the semantic traffic management provides a "buffer" until the traffic load is lowered otherwise, e.g. by admission control schemes, dropping some flows, or invoking a hand-off of some terminals.

\section{References}

1. P. Bhagwat, P. Bhattacharya, A. Krishna, and S. Tripathi, "Enhancing throughput over wireless LANs using channel state dependent packet scheduling," in IEEE INFOCOM, 1994.

2. M. Smirnov, E. Biersack, C. Blondia, O. Bonaventure et al., Quality of future Internet services, Springer-Verlag, 2003.

3. T. Le-Ngoc, N. Damji, and Y. Xu, "Dynamic resource allocation for multimedia services over OFDM downlink in cellular systems," in Proc. of IEEE Vehicular Technology Conference Spring, 2004.

4. M. Hemy, P. Steenkiste, and T. Gross, "Evaluation of adaptive filtering of MPEG system streams in IP networks," in Proc. of IEEE International Conference on Multimedia \& Expo, 2000.

5. C. Fraleigh, S. Moon, B. Lyles, C. Cotton et al., "Packet-level traffic measurements from the sprint IP backbone," IEEE Network, vol. 17, 2003.

6. S. Shakkottai and A. Stolyar, "Scheduling algorithms for a mixture of realtime and non-real-time data in HDR," in Proc. of 17th International Teletraffic Congress, 2001.

7. J. Gross, H. Karl, F. Fitzek, and A. Wolisz, "Comparison of heuristic and optimal subcarrier assignment algorithms," in Proc. of International Conference on Wireless Networks, 2003.

8. J. Gross, J. Klaue, H. Karl, and A. Wolisz, "Cross-layer optimization of OFDM transmission systems for MPEG-4 video streaming," Computer Communications, vol. 27, 2004.

9. A. Aguiar and J. Gross, "Wireless channel models," Technical Report TKN03-007, Telecommunication Networks Group, April 2003.

10. J. Medbo and P. Schramm, "Channel models for HIPERLAN/2," document no. 3ERI085B, ETSI/BRAN, 1998.

11. A. Reyes-Lecuona, E. González-Parada, E. Casilari, J. C. Casasola et al., "A page-oriented WWW traffic model for wireless system simulations," in Proc. of 16th International Teletraffic Congress, 1999. 